\title{
Temporal evolution of a radiating star via Lie symmetries
}

\author{
Andronikos Paliathanasis ${ }^{1,2, a}$, Megandhren Govender ${ }^{3, b}$, Genly Leon ${ }^{4, c}$ \\ ${ }^{1}$ Institute of Systems Science, Durban University of Technology, Durban, South Africa \\ 2 Instituto de Ciencias Físicas y Matemáticas, Universidad Austral de Chile, 5090000 Valdivia, Chile \\ ${ }^{3}$ Department of Mathematics, Faculty of Applied Sciences, Durban University of Technology, Durban, South Africa \\ ${ }^{4}$ Departamento de Matemáticas, Universidad Católica del Norte, Avda. Angamos 0610, Casilla 1280, Antofagasta, Chile
}

Received: 12 January 2021 / Accepted: 3 August 2021 / Published online: 10 August 2021

(C) The Author(s) 2021

\begin{abstract}
In this work we present for the first time the general solution of the temporal evolution equation arising from the matching of a conformally flat interior to the Vaidya solution. This problem was first articulated by Banerjee et al. (Phys Rev D 40:670, 1989) in which they provided a particular solution of the temporal equation. This simple exact solution has been widely utilised in modeling dissipative collapse with the most notable result being prediction of the avoidance of the horizon as the collapse proceeds. We study the dynamics of dissipative collapse arising from the general solution obtained via the method of symmetries and of the singularity analysis. We show that the end-state of collapse for our model is significantly different from the widely used linear solution.
\end{abstract}

\section{Introduction}

The end state of a star resulting from continued gravitational collapse is still a much debated topic in relativistic astrophysics. It has been shown in some classes of models that shear plays an important role in producing naked singularities. On the other hand, the absence of shear during gravitational collapse of reasonable matter distributions (for example in the case of perfect fluids) always results in a black hole [2]. An interesting study of gravitational collapse in the shear-free regime was proposed by Banerjee, Chaterjee and Dadhich (hereafter referred to as the $B C D$ model [3]) in which the collapse proceeds without the formation of the horizon. The so-called horizon-free collapse model has been explored in various contexts including higher dimensional spacetimes, Euclidean stars and class-one spacetimes [4-8].

\footnotetext{
a e-mail: paliathanasis@gmail.com

b e-mail: megandhreng@dut.ac.za (corresponding author)

c e-mail: genly.leon@ucn.cl
}

In this work we explore the boundary condition arising from the matching of a spherically symmetric imperfect fluid configuration undergoing dissipative collapse and matched to a Vaidya atmosphere. The boundary condition describing conservation of momentum flux across the bounding hypersurface is a second order nonlinear differential equation governing the temporal behaviour of the model. A particular solution of this equation which is linear in time has been widely used to model dissipative collapse in which the horizon never forms. We utilise a symmetry approach to generate the general solution of the boundary condition. We explore the physics associated with the collapsing core, particularly during the late stages of collapse.

This paper is structured as follows. In Sect. 2 we present the field equations which govern the interior spacetime of the collapsing sphere and the junction conditions required for the smooth matching of the interior spacetime to the Vaidya exterior. We study the temperature profiles of the collapsing star in Sect. 3. A physical analysis of the thermodynamical variables and time of formation of the horizon is carried out in Sect. 4. We conclude with an overall discussion in Sect. 5. For completeness we add Appendix A. In Appendix A.1 is presented the stability analysis of powerlaw exact solutions. Appendix A.2 is devoted to symmetries and singularity analysis.

\section{Dissipative collapse}

When modeling a radiating star undergoing shear-free gravitational collapse the interior spacetime is described by the spherically symmetric line element [9]

$d s^{2}=-A^{2}(r, t) d t^{2}+B^{2}(r, t)\left[d r^{2}+r^{2} d \theta^{2}+r^{2} \sin ^{2} \theta d \phi^{2}\right]$, 
in which the metric functions $A$ and $B$ are yet to be determined. The energy momentum tensor for the interior matter distribution is described by an imperfect fluid given by

$$
T_{a b}=(\rho+p) u_{a} u_{b}+p g_{a b}+q_{a} u_{b}+q_{b} u_{a} .
$$

where $\rho$ and $p$ are the fluid energy density and pressure. The heat flow vector $q^{a}$ is orthogonal to the velocity vector so that $q^{a} u_{a}=0$. The Einstein field equations governing the interior of the stellar fluid is

$$
\begin{aligned}
\rho= & 3 \frac{1}{A^{2}} \frac{B_{t}^{2}}{B^{2}}-\frac{1}{B^{2}}\left(2 \frac{B_{r r}}{B}-\frac{B_{r}^{2}}{B^{2}}+\frac{4}{r} \frac{B_{r}}{B}\right), \\
p= & \frac{1}{A^{2}}\left(-2 \frac{B_{t t}}{B}-\frac{B_{t}^{2}}{B^{2}}+2 \frac{A_{t}}{A} \frac{B_{t}}{B}\right) \\
& +\frac{1}{B^{2}}\left(\frac{B_{r}{ }^{2}}{B^{2}}+2 \frac{A_{r}}{A} \frac{B_{r}}{B}+\frac{2}{r} \frac{A_{r}}{A}+\frac{2}{r} \frac{B_{r}}{B}\right), \\
p= & -2 \frac{1}{A^{2}} \frac{B_{t t}}{B}+2 \frac{A_{t}}{A^{3}} \frac{B_{t}}{B}-\frac{1}{A^{2}} \frac{B_{t}{ }^{2}}{B^{2}}+\frac{1}{r} \frac{A_{r}}{A} \frac{1}{B^{2}} \\
& +\frac{1}{r} \frac{B_{r}}{B^{3}}+\frac{A_{r r}}{A} \frac{1}{B^{2}}-\frac{B_{r}{ }^{2}}{B^{4}}+\frac{B_{r r}}{B^{3}}, \\
Q= & -\frac{2}{A B}\left(-\frac{B_{r t}}{B}+\frac{B_{r} B_{t}}{B^{2}}+\frac{A_{r}}{A} \frac{B_{t}}{B}\right),
\end{aligned}
$$

where $Q=\left(q_{a} q^{a}\right)^{1 / 2}$ is the magnitude of the heat flux. We obtain the condition of pressure isotropy by equating (4) and (5)

$0=\frac{1}{B^{2}}\left[\frac{A_{r r}}{A}+\frac{B_{r r}}{B}-\left(2 \frac{B_{r}}{B}+\frac{1}{r}\right)\left(\frac{A_{r}}{A}+\frac{B_{r}}{B}\right)\right]$.

This equation has been solved under various assumptions and transformations. It was Ivanov [10] who observed that if the constants of integration of a static solution to the pressure isotropy condition in comoving and isotropic coordinates are allowed to evolve with time, then, the time-dependent 'solution' will automatically satisfy (7). Shear-free, radiating fluids in the presence of bulk viscosity were widely explored by Sussman. Starting with the most general spherically symmetric, shear-free metric, Sussman obtained a large family of exact solutions of the Einstein field equations which included Petrov type D, conformally flat and self-similar solutions [11]. In particular, he showed that the constraints arising from the field equations for shear-free spacetimes can be treated as a linear algebraic system on the derivatives of the metric potentials.

Since the star is radiating energy, the exterior spacetime is described by the Vaidya metric [12]

$$
\begin{aligned}
d s^{2}= & -\left(1-\frac{2 m(v)}{\mathrm{r}}\right) d v^{2}-2 d v d \mathrm{r}+\mathrm{r}^{2}\left[d \theta^{2}\right. \\
& \left.+\mathrm{r}^{2} \sin ^{2} \theta d \phi^{2}\right]
\end{aligned}
$$

where $v$ is the retarded time and $m$ is the total mass inside the comoving surface $\Sigma$ forming the boundary of the star. The necessary junction conditions for the smooth matching of the interior line element (1) to the exterior spacetime (8) was first obtained by Santos [13] and has been succinctly presented here for easy reference

$$
\begin{aligned}
(r B)_{\Sigma} & =\mathrm{r}_{\Sigma}, \\
p_{\Sigma} & =\left(q^{1} B\right)_{\Sigma}, \\
m_{\Sigma} & =\left[\frac{r^{3} B \dot{B}^{2}}{2 A^{2}}-r^{2} B^{\prime}-\frac{r^{3} B^{\prime 2}}{2 B}\right]_{\Sigma},
\end{aligned}
$$

where $m_{\Sigma}$ is the total mass within a sphere of radius $r_{\Sigma}$ and (10) represents the conservation of the momentum flux across the boundary $\Sigma$.

An interesting approach to dissipative collapse is to explore the non-formation of the horizon in which the collapse rate is balanced by the rate at which energy is radiated to the exterior spacetime. The trio Banerjee, Chaterjee and Dadhich studied such a scenario by considering a simple radiative model in which the metric ansatz assumed is

$A=1+\zeta_{0} r^{2}$,

$B=R(t)$

where $\zeta_{0}$ and $C$ are positive constants. The collapse evolves from $t=-\infty$ until $t=0$. Utilising the above ansatz together with (4) and (6) in (10) we obtain

$2 R \ddot{R}+\dot{R}^{2}+\alpha \dot{R}=\beta$

where $\alpha$ and $\beta$ are constants. A special and simple solution to this equation is $R=-C t$ where $C>0$ is a constant. Since the star is collapsing, we require the expansion scalar, $\Theta=\frac{3}{A} \frac{\dot{R}}{R}<0$. This solution first made its appearance in the literature in 1989 when Banerjee et al. [1] presented the most general class of conformally flat radiating solutions. While the solution has a simple form it is remarkable that it has revealed rich and diversified toy models of dissipative collapse. It has been observed in the $B C D$ paper that the ratio

$1-\frac{2 m_{\Sigma}}{r_{\Sigma}}$

is independent of time. This can be easily seen from Eqs. (9) and (11) in which both the area radius and mass are in linear in $t$. Thus the ratio of mass to area radius is independent of time and the boundary surface cannot reach the horizon.

\subsection{A new radiating model}

We now model a radiating star undergoing dissipative collapse by digressing from the simple linear time-dependence of $B(r, t)$. To this end we utilise the truncated solution (A.6) 
reported in Appendix A.2 and derived by using the Lie symmetry analysis,

$R(t)=R_{0} t^{2 / 3}-\frac{3 \alpha}{4} t$

with $\beta=-\frac{3 \alpha^{2}}{4}$. The Einstein field equations (3)-(6) reduce to

$$
\begin{aligned}
\rho & =\frac{\left(8 R_{0}-9 \alpha t^{1 / 3}\right)^{2}}{3 t^{2}\left(-4 R_{0}+3 \alpha t^{1 / 3}\right)^{2}\left(1+\zeta_{0} r^{2}\right)^{2}} \\
p & =\frac{32 \alpha R_{0}+3 t^{1 / 3}\left(-9 \alpha^{2}+64 \zeta_{0}\left(1+r^{2} \zeta_{0}\right)\right)}{3 t^{5 / 3}\left(4 R_{0}-3 \alpha t^{1 / 3}\right)^{2}\left(1+\zeta_{0} r^{2}\right)^{2}} \\
q B & =-\frac{55\left(-32 \alpha R_{0}+27 \alpha^{2} t^{1 / 3}-192 \zeta_{0} t^{1 / 3}\left(1+\zeta_{0} r^{2}\right)\right)}{3 t^{5 / 3}\left(-4 R_{0}+3 \alpha t^{1 / 3}\right)^{2}\left(1+\zeta_{0} r^{2}\right)^{2}} .
\end{aligned}
$$

We also calculate the mass function and luminosity at infinity to be

$$
\begin{gathered}
m=\frac{r^{2}\left(8 R_{0}-9 \alpha t^{1 / 3}\right)^{2}\left(4 R_{0}-3 \alpha t^{1 / 3}\right)}{1152\left(1+\zeta_{0} r^{2}\right)^{2}}, \\
L_{\infty}=\frac{\alpha r_{0}\left(32 R_{0}-27 \alpha t^{1 / 3}\right)\left(8 R_{0}-9 \alpha t^{1 / 3}\right) \Xi}{864 t^{7 / 3}\left(4 R_{0}-3 \alpha t^{1 / 3}\right)^{2}\left(1+\zeta_{0} r_{0}^{2}\right)^{4}}
\end{gathered}
$$

where we have defined

$$
\Xi=8 R_{0} r_{0}+3 t^{1 / 3}\left(4-3 \alpha r_{0}+4 \zeta_{0} r_{0}^{2}\right)
$$

and $r=r_{0}$ defines the boundary of the star at some fixed time.

\subsection{The role of an equation of state}

There have been many attempts at incorporating an equation of state $(\mathrm{EoS})$ in radiating stellar models. This is an important requirement which relates the pressure to the fluid density and this in turn makes the interplay between these thermodynamical quantities transparent. An early attempt at imposing a linear equation of state of the form, $p_{r}=\gamma \rho$ where $\gamma$ is a constant was carried out by Wagh et al. [34] in which they matched a shear-free interior metric to the Vaidya exterior. In their model the temporal dependence was linear. More recently, Bogadi et al. [35] attempted to model a radiating star which collapses from an initial static configuration. In this work, both the static and dynamical models obey a linear EoS. In order to satisfy the boundary condition, it was shown that the EoS parameter, $\gamma=-1 / 3$. This investigation demonstrated the difficulty of imposing an EoS for the dynamical model, i. e., the gravitational potentials must simultaneously satisfy the EoS at each interior point $(r, t)$ of the collapsing body and the boundary condition (where $r=r_{0}$, is constant). At best, one can choose an initial static configuration which satisfies a given linear EoS. This choice automatically changes the dynamical boundary condition as the radial pressure vanishes at the boundary of the static configuration. In our work, the metric potentials $(A, B)$ given by (12) and (13) do not describe an initial static configuration. At the very least the simple forms chosen for $(A, B)$ here could mimic an EoS in the appropriate limit but this carries no guarantee until the boundary condition is satisfied. In the BCD model the energy density and pressure both diverge as $1 / t^{2}$ and the mass evolves linearly with time. In addition, the ratio $p / \rho$ is independent of time and increases as $r^{2}$. In our model $p / \rho$ increases as times evolves and changes as $1 / r^{3}$. It is clear that relationship between pressure and density is significantly altered when higher order terms are included in the temporal behaviour of the model.

\section{Causal heat flow}

The role of causal heat flow during dissipative collapse has been extensively studied by Herrera and co-workers [36-38] and references therein. It has been demonstrated that relaxational effects lead to higher core temperatures as the collapse proceeds with cooling being enhanced in the surface layers. The noncausal Eckart formalism may hold during an epoch when the fluid is close to hydrostatic equilibrium. However, as the collapse proceeds the noncausal nature of the Eckart framework leads to infinite propagation speeds of the thermal signals and unstable equilibrium states. Earlier work by Di Prisco et al. [36] has shown that relaxational effects impact on the luminosity profiles of radiating stars. In order to study the impact of relaxation times on the temperature profiles we adopt a causal heat transport equation of Maxwell-Cattaneo form [30]

$\tau h_{a}{ }^{b} \dot{q}_{b}+q_{a}=-\kappa\left(D_{a} T+T \dot{u}_{a}\right)$,

where the relaxation time is given by

$\tau=\kappa T \beta$

for the heat flux. The appearance of the relaxation time restores causality and has been successful in modelling highfrequency phenomena in electronics and fluid flow [31]. For the line element (1) the causal heat transport equation (23) becomes

$\tau(q B)_{, t}+A(q B)=-\kappa \frac{(A T)_{, r}}{B}$,

which governs the behavior of the temperature. Setting $\tau=0$ in (25) we obtain the familiar Fourier heat transport equation

$A(q B)=-\kappa \frac{(A T)_{, r}}{B}$,

which predicts reasonable temperatures when the fluid is close to quasi-stationary equilibrium. 
Following the work of [32] we adopt the following thermodynamic coefficients for radiative transfer where we assume that heat is being carried away from the core via thermally generated neutrinos. The thermal conductivity assumes the form

$\kappa=\chi T^{3} \tau_{\mathrm{c}}$,

where $\chi(\geq 0)$ is a constant and $\tau_{\mathrm{c}}$ represents the mean collision time between massless and massive particles. Martinez [33] has shown that $\tau_{\mathrm{c}} \propto T^{-3 / 2}$ for thermally generated neutrinos within the core of neutron stars. To this end we assume

$\tau_{\mathrm{c}}=\left(\frac{\psi}{\chi}\right) T^{-\omega}$

where $\psi(\geq 0)$ and $\omega(\geq 0)$ are constants and for $\omega=\frac{3}{2}$ we regain the treatment due to Martinez. We observe that this form implies that the mean collision time decreases with an increase in temperature, as expected. We assume that relaxation time is proportional to the collision time:

$\tau=\left(\frac{\lambda \chi}{\psi}\right) \tau_{\mathrm{c}}$,

where $\tau(\geq 0)$ is a constant. This assumption may hold for a limited epoch of the collapse process. Putting all together in (25) we obtain

$\lambda(q B){ }_{, t} T^{-\omega}+A(q B)=-\psi \frac{T^{3-\omega}(A T)_{, r}}{B}$.

It has been shown that in the case $\omega=0$ (which corresponds to constant mean collision time), the causal transport equation (30) yields the following temperature profile [39]

$$
(A T)^{4}=-\frac{4}{\psi}\left[\lambda \int A^{3} B(q B){ }_{, t} \mathrm{~d} r+\int A^{4} q B^{2} \mathrm{~d} r\right]+\mathscr{F}(t)
$$

where $\mathscr{F}(t)$ is an integration function. We can evaluate $\mathcal{F}(t)$ by recalling that the effective surface temperature of a star is given by

$$
\left(T^{4}\right)_{\Sigma}=\left(\frac{1}{r^{2} B^{2}}\right)_{\Sigma}\left(\frac{L_{\infty}}{4 \pi \sigma}\right)_{\Sigma}
$$

where $\delta(>0)$ is a constant. For our model, we are able to complete the integration in (31) and the temperature is plotted in Fig. 6.

\section{Physical analysis}

In order to establish the physical viability of our model, we have plotted the density, pressure, heat flow and the energy conditions, respectively as functions of the radial and temporal coordinates in Figs. 1, 2, 3, 4, 5 and 6 . We observe that the density (Fig. 1) and pressure (Fig. 2) are monotonically decreasing functions of the radial coordinate. Furthermore, as the collapse proceeds, the density and pressure increase. This is expected as the core collapses the matter gets squeezed into smaller volumes thus increasing the density and the pressure within the smaller sphere. In the case of the BCD model, the rate at which the body radiates energy is balanced by the rate at which it collapses which leads to the final 'evaporation' of the star. It is worth pointing out that the luminosity as given by (21) vanishes when

$t_{(b h)_{1}}=0.702332 v^{3}$,

$t_{(b h)_{2}}=1.66479 v^{3}$,

$t_{(b h)_{3}}=-18.963 \frac{\alpha^{3} v^{3}}{\left(4-3 r_{0} \alpha+4 r_{0}^{2} \zeta_{0}\right)^{3}}$,

where we have defined $v=\frac{R_{0}}{\alpha}$. Since the collapse proceeds from $-\infty<t<0$, we must have $t_{(b h)_{i}}<0$ if we want the horizon to form before the collapse ends. For $\alpha>0$ we must $R_{0}<0$ which ensures that $t_{(b h)_{1}}$ and $t_{(b h)_{2}}$ are both negative. For $t_{(b h)_{3}}<0$ we must have $4-3 r_{0} \alpha+4 r_{0}^{2} \zeta_{0}$ which places a restriction on $\zeta_{0}$. In the BCD model where the horizon never forms, no such restriction is placed on $\zeta_{0}$. Figure 3 shows that the heat flux (energy output) increases with time. As the density increases, the thermonuclear processes become more efficient resulting in higher energy outputs for late times. The mass profile is depicted in Fig. 4. The mass of the sphere increases monotonically from the center to the boundary. The mass decreases with time as star radiates energy as it collapses. Figures 5 and 6 confirm that the energy conditions required for a realistic stellar core are obeyed everywhere inside the star. We have plotted the causal temperature profiles in Fig. 7. The blue curve represents the BCD temperature profile whereas the orange curve describes the evolution of the temperature as a function of the radial coordinate for our nonlinear solution. It is evident that the nonlinear solution predicts higher core temperatures than the linear BCD model. At this juncture it is necessary to point out that the condition of pressure isotropy which we employed to generate the static solution is unstable in the sense that such an isotropic matter distribution will evolve into an anisotropic regime as it leaves hydrostatic equilibrium. The presence of dissipative fluxes (in the form of heat flow), density inhomogeneities and/or nonzero shear within the fluid flow, can disrupt the condition of pressure isotropy leading to unequal radial and tangential stresses within the collapsing fluid [40]. Our aim of this work was to demonstrate the existence of the general solution of the junction condition which encodes the temporal behaviour of the model. We observe that the dynamical behaviour (temperature profiles and horizon formation) are clearly different from the simple linear temporal evolution assumed in the BCD model. We have shown that the inclusion of higher order terms in the temporal evolution of the model significantly affects the dynamics of the collapse 


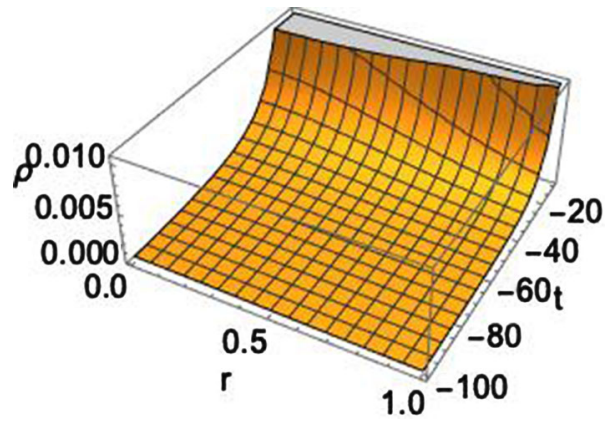

Fig. 1 Density as a function of the radial and temporal coordinates

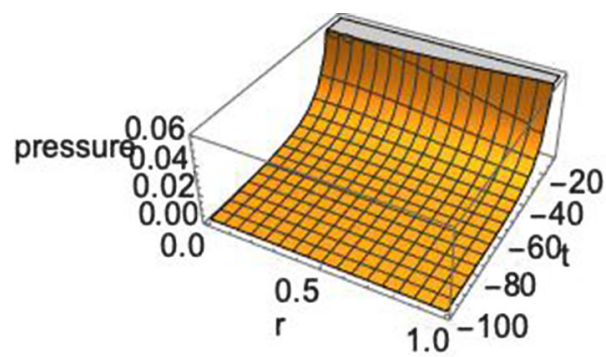

Fig. 2 Pressure as a function of the radial and temporal coordinates

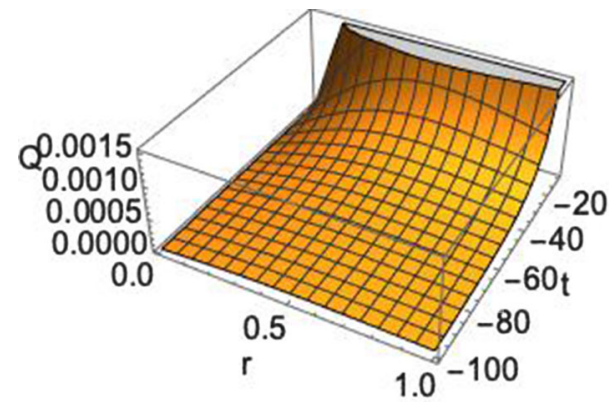

Fig. 3 Heat flow as a function of the radial and temporal coordinates

process. This can be seen in the altered temperature distribution at each interior point of the collapsing fluid and the time of formation of the horizon.

\section{Concluding remarks}

In this exposition we analysed a differential equation arising from the modeling of a star undergoing dissipative collapse. We have presented the general solution to the boundary condition for a particular type of shear-free, dissipative collapse. In this model the gravitational potentials are separable in the radial and temporal coordinates and the pressure is isotropic at each interior point of the collapsing distribution. We carried out an extensive stability analysis of the solution arising from the temporal equation in Appendix A.1. We have analytically proved the following results: (i) The solution of (14) $R=-C t$ defined for $-\infty<t \leq 0$, where $C>0$ is a fixed

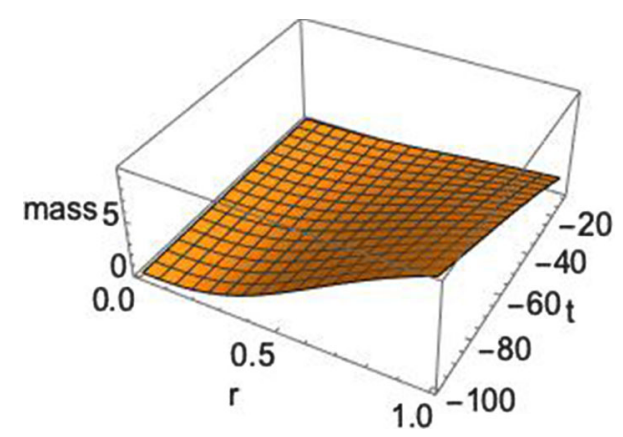

Fig. 4 Mass profile as a function of the radial and temporal coordinates

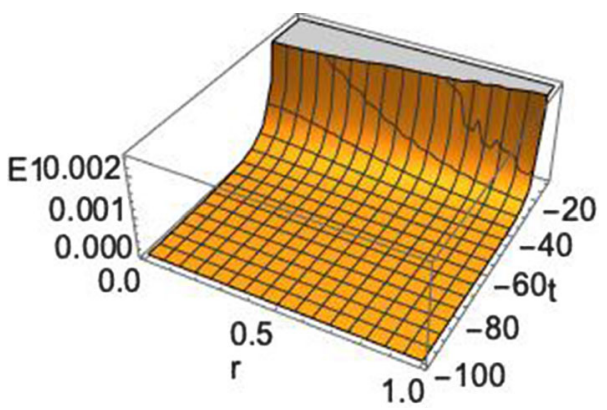

Fig. $5 E 1=(\rho+p)^{2}-4 Q^{2}>0$ as a function of the radial and temporal coordinates

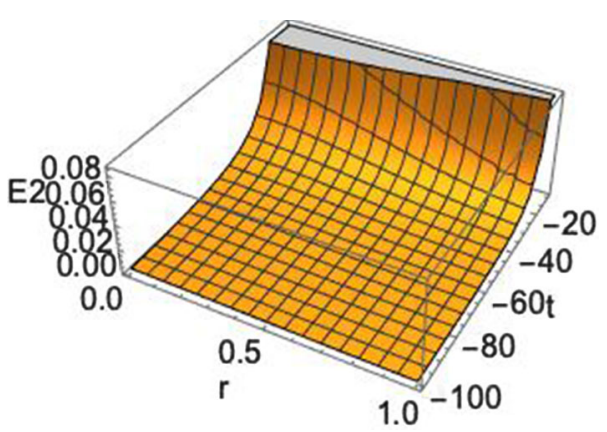

Fig. $6 E 2=\rho-3 p+\left[(\rho+p)^{2}-4 Q^{2}\right]^{1 / 2}>0$ as a function of the radial and temporal coordinates

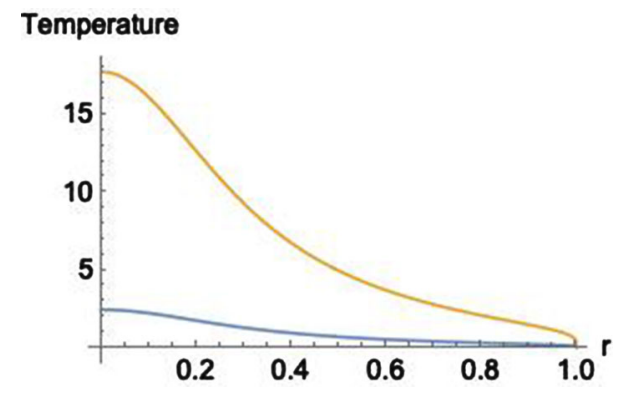

Fig. 7 Temperature profiles as a function of the radial coordinate 
constant by Banerjee et al. [1], is unstable as $t \rightarrow 0^{-}$and stable as $t \rightarrow-\infty$. (ii) We found a family of solutions of (14) given by

$R(t)=\frac{\alpha c_{1}}{2-\theta^{*}}+\frac{\alpha\left(\theta^{*}-1\right) t}{2-\theta^{*}}, \quad c_{1} \neq 0$,

parametrised by $-\infty<\theta^{*}<\infty$. They are defined in the semi-infinite-interval $-\infty<t \leq 0$, and are stable as $t \rightarrow$ $0^{-}$. Our solutions are intrinsically different from the closedform solution of Banerjee et al. [1] in terms of their stability as well as in nature, given that $\lim _{t \rightarrow 0^{-}} R(t)=\frac{\alpha c_{1}}{2-\theta^{*}} \neq 0$, as a difference with the closed-form solution of Banerjee et al. [1] which satisfies $\lim _{t \rightarrow 0^{-}} R(t)=0$. The affine parameter $\frac{\alpha c_{1}}{2-\theta^{*}}$ makes a subtle difference concerning stability as $t \rightarrow 0^{-}$. We showed that a particular nonlinear temporal dependence produces drastically different physics from the linear model. This is an important point to note, albeit that the collapsing sphere described here represents a toy model of dissipative collapse.

Our model does not feature an EoS as the chosen metric functions are simplistic in nature. An inclusion of an EoS will form the basis of future work.

Acknowledgements AP and GL were funded by Agencia Nacional de Investigación y Desarrollo-ANID through the program FONDECYT Iniciación grant no. 11180126. Additionally, GL thanks the support of Vicerrectoría de Investigación y Desarrollo Tecnológico at Universidad Católica del Norte. We thank the anonymous referee for his/her valuable comments which have helped us improve our work.

Data Availability Statement This manuscript has no associated data or the data will not be deposited. [Authors' comment: The work contained in this article is based on theoretical computation and as such no experimental data has been generated. All data generated in this study are self-contained in this article. So there is no data to be deposited.]

Open Access This article is licensed under a Creative Commons Attribution 4.0 International License, which permits use, sharing, adaptation, distribution and reproduction in any medium or format, as long as you give appropriate credit to the original author(s) and the source, provide a link to the Creative Commons licence, and indicate if changes were made. The images or other third party material in this article are included in the article's Creative Commons licence, unless indicated otherwise in a credit line to the material. If material is not included in the article's Creative Commons licence and your intended use is not permitted by statutory regulation or exceeds the permitted use, you will need to obtain permission directly from the copyright holder. To view a copy of this licence, visit http://creativecomm ons.org/licenses/by/4.0/.

Funded by SCOAP ${ }^{3}$.
Appendix A: Dynamical systems analysis, symmetries and singularity analysis

Appendix A.1: Stability analysis of exact solutions

We have the main equation (14) where $\alpha$ and $\beta$ are constants. For convenience we assume $\alpha>0$. A special and simple solution to this equation is $R=-C t$ defined for $-\infty<t \leq$ 0 , where $C>0$ is a constant given by the positive root of $-\beta+C^{2}-\alpha C=0$.

For analysis of the stability of the scaling solution $R_{S}(t)=$ $-C t$, with $C=\frac{1}{2}\left(\sqrt{\alpha^{2}+4 \beta}+\alpha\right)>0$ in the interval $-\infty<t<0$ we use similar methods as in Liddle and Scherrer [14] and in Uzan [15]. Defining the new time variable $\eta$ through $t=-e^{-\eta},-\infty<\eta<\infty$ such that $t \rightarrow-\infty$ as $\eta \rightarrow-\infty$ and $t \rightarrow 0$ as $\eta \rightarrow+\infty$, as well as the variables $\varepsilon(\eta)=\frac{R(\eta)}{R_{s}(\eta)}-1, \quad v(\eta)=\varepsilon^{\prime}(\eta)$ in which $R(\eta)=R\left(-e^{-\eta}\right)$ and $R_{S}(\eta)=-C t(\eta)=C e^{-\eta}$ allows the scaling solution to be translated to the origin. Finally, we obtain the system

$\varepsilon^{\prime}=v$,

$v^{\prime}=\frac{\varepsilon(\alpha-C \varepsilon-2 C)}{2 C(\varepsilon+1)}+v\left(2-\frac{\alpha}{2 C \varepsilon+2 C}\right)-\frac{v^{2}}{2(\varepsilon+1)}$,

with linearisation matrix $J(\varepsilon, v)$. The latter system admits the stationary points $P_{1}=(0,0)$ and $P_{2}=\left(\frac{\alpha}{C}-2,0\right)$. At point $P_{1}$, the matrix $J(0,0)$ has eigenvalues $\left\{1,1-\frac{\alpha}{2 C}\right\}$. Assume first $\beta \geq 0$. In this case, the origin is always unstable as $\eta \rightarrow$ $+\infty$ due to $2 C=\alpha+\sqrt{\alpha^{2}+4 \beta}>\alpha$. The origin is stable as $\eta \rightarrow-\infty$. Moreover, at the point $P_{2}$ we find that the matrix $J\left(\frac{\alpha}{C}-2,0\right)$ has eigenvalues $\left\{1, \frac{2 C-\alpha}{2(C-\alpha)}\right\}$. Due to $2 C-\alpha>0$ it is unstable as $\eta \rightarrow \infty$. Indeed for $0<\frac{\alpha}{2}<C<\alpha$ it is a saddle, whereas for $C>\alpha>0$ is an unstable node. The last condition is forbidden due to the physical condition $\varepsilon \geq-1$ evaluated at $(\varepsilon, v)=\left(\frac{\alpha}{C}-2,0\right)$ implies $\alpha \geq C$. In the case $\beta<0, \alpha<-2 \sqrt{-\beta}$ or $\beta<0, \alpha>2 \sqrt{-\beta}$ , we have two solutions $R_{s \pm}(\eta)=-C_{ \pm} t(\eta)=C_{ \pm} e^{-\eta}$, where $2 C_{ \pm}=\alpha \pm \sqrt{\alpha^{2}-4|\beta|}$. Observe that $2 C_{+}>\alpha$, implies that $R_{S+}(\eta)$ is an unstable solution (unstable node) as $\eta \rightarrow \infty$. Due to $\alpha>2 C_{-}>0, R_{S_{-}}(\eta)$ is an unstable (saddle) solution.

On the other hand, there are non-trivial dynamics as $(\varepsilon, v)$ are unbounded. Let us now define $\phi:=h(\varepsilon)=(\varepsilon+1)^{-1 / n}$, $\theta=v-\varepsilon$, where the coordinate transformation $\phi=h(\varepsilon)$ for $n>1$ maps the interval $\left[\varepsilon_{0}, \infty\right)$ onto $(0, \delta]$, with $\delta=h\left(\varepsilon_{0}\right)$, satisfying $\lim _{\varepsilon \rightarrow+\infty} h(\varepsilon)=0$. We then obtain the dynamical system

$$
\phi^{\prime}=-\frac{\phi}{n}+\left(\frac{1}{n}-\frac{\theta}{n}\right) \phi^{n+1}
$$




$$
\theta^{\prime}=\phi^{n}\left(\left(1-\frac{\alpha}{2 C}\right) \theta-\frac{\theta^{2}}{2}\right)
$$

where $2 C-\alpha>0$ and $0<\phi<\delta^{1 / 2}, \theta \in K$, where $K$ is a compact set. Neglecting higher order terms $\propto \phi^{n+1}$ as $\phi \rightarrow 0$ in Eq. (A.3) we obtain the asymptotic solution: $\phi(\eta)=e^{-\frac{\eta}{n}} c_{1}, \theta(\eta)=\frac{(2 C-\alpha)}{C-\exp \left(\frac{(2 C-\alpha)\left(e^{-\eta} c_{1}^{n}+2 C c_{2}\right)}{2 C}\right)}$. The system (A.3), (A.4) admits a line of fixed points $L:(\phi, \theta)=$ $\left(0, \theta^{*}\right)$ as $\eta \rightarrow \infty$ for bounded $\theta$. As $\eta \rightarrow \infty$ the eigenvalues associated to $L$ are $\left\{-\frac{1}{n}, 0\right\}$. Therefore, it is normally hyperbolic and stable. It is verified that asymptotic solution showed before converges to it.

A summary of our analytical findings indicate: (i) The solution of (14) $R=-C t$ defined for $-\infty<t \leq 0$, where $C>0$ is a fixed constant, is unstable as $t \rightarrow 0^{-}$and stable as $t \rightarrow-\infty$. (ii) The curve of fixed points $L:(\phi, \theta)=\left(0, \theta^{*}\right)$ (i.e., $\varepsilon \rightarrow \infty$, and $v \rightarrow \infty$, in such a way that $v-\varepsilon \rightarrow \theta^{*}$ ) is stable as $\eta \rightarrow \infty$ for bounded $\theta$. Result (ii) means that, as $\eta \rightarrow \infty$, we have $\varepsilon^{\prime}(\eta)=\varepsilon(\eta), \varepsilon(\infty)=\infty$. Hence, $\varepsilon(\eta)=$ $c_{1} e^{\eta}-\theta^{*}, c_{1} \neq 0$. Then, $R(\eta)=C\left(c_{1}-\left(\theta^{*}-1\right) e^{-\eta}\right)$.

Substituting into (14), we find the constraint $C \theta^{*}(\alpha+$ $\left.C\left(\theta^{*}-2\right)\right)=0$. We have some specific solutions when $\theta^{*} \in\left\{0,2-\frac{\alpha}{C}\right\}$. Recall that $\theta^{*}$ is an arbitrary constant value by definition of line $L$, so the natural condition is $C=\frac{\alpha}{2-\theta^{*}}$. Then, the solution of (14) given by (36) defined in the semiinfinite-interval $-\infty<t \leq 0$, is stable as $t \rightarrow 0^{-}(\eta \rightarrow$ $+\infty)$. Finally, $\lim _{t \rightarrow 0^{-}} R(t)=\frac{\alpha c_{1}}{2-\theta^{*}} \neq 0$ by construction. We should point out that the in(stability) of the solutions demonstrated in this section is not related to the instability of the pressure isotropy condition highlighted in [40].

Appendix A.2: Symmetries and singularity analysis

Let us now turn our attention to the boundary condition (14). Equation (14) is invariant under the infinitesimal transformation [16] $\bar{t} \rightarrow t+\varepsilon\left(\alpha_{1}+\alpha_{2} t\right), \bar{R} \rightarrow R+\varepsilon\left(\alpha_{2} R\right)$ where $\varepsilon$ is an infinitesimal parameter. We infer that Eq. (14) admits as Lie point symmetries the elements of the two-dimensional Lie algebra $\left\{\partial_{t}, t \partial_{t}+R \partial_{R}\right\}$ which form the $A_{2,2}$ Lie algebra in the Morozov-Mubarakzyanov classification scheme [2225]. The application of the Lie symmetries in gravitational physics has provided us with many interesting results, for instance see [17-21] and references therein.

From the vector field $\partial_{t}$ we find the reduced equation $2 x y \frac{d y(x)}{d x}+y^{2}(x)+\alpha y(x)-\beta=0$ with $y(x)=\dot{R}, x=R$. The reduced equation gives

$$
\begin{aligned}
\ln \left(x-x_{0}\right)= & -\ln \left(y^{2}+\alpha y-\beta\right) \\
& -\frac{2 \alpha}{\sqrt{\alpha^{2}+4 \beta}} \operatorname{arctanh}\left(\frac{2 y+\alpha}{\sqrt{\alpha^{2}+4 \beta}}\right) .
\end{aligned}
$$

or in the special case where $\beta=-\frac{\alpha^{2}}{4}$, the reduced equation provides $\ln (2+y+\alpha)+\frac{\alpha}{2 y+\alpha}=-\frac{1}{2} \ln \left(x-x_{0}\right)$.

On the other hand, from the Lie symmetry $t \partial_{t}+R \partial_{R}$ for Eq. (14) it follows $2 z(y(z)-z) \frac{d y(z)}{d z}+y^{2}(z)+\alpha y(z)-\beta=$ 0 . This is an Abel equation and can be integrated by using a Lie integration factor. A special solution is the constant value of $y=y_{0}$ with $y_{0}^{2}+\alpha y_{0}-\beta=0$, however, such solution leads to the closed-form solution of Banerjee et al. [1].

We proceed with our analysis by writing a closed form solution of Eq. (14) derived with the singularity analysis. The modern treatment of the singularity analysis is described by the ARS algorithm [26-29]. For Eq. (14) the leading-order term is found to be $R_{\text {leading }}(t)=R_{0}\left(t-t_{0}\right)^{\frac{2}{3}}$, where $t_{0}$ indicates the location of the movable singularity and $R_{0}$ is arbitrary. The resonances, are derived to be $s_{1}=-1$ and $s_{4}=4$, which means that the analytic solution of (14) can be expressed in terms of the Right Painlevé Series

$$
\begin{aligned}
R(t)= & R_{0}\left(t-t_{0}\right)^{\frac{2}{3}}+R_{1} t+R_{2}\left(t-t_{0}\right)^{\frac{4}{3}} \\
& +R_{3}\left(t-t_{0}\right)^{\frac{5}{3}}+\cdots .
\end{aligned}
$$

We replace in (14) from where we find that $R_{1}=-\frac{3 \alpha}{4}, R_{2}=$ $\frac{9}{320 R_{0}}\left(3 \alpha^{2}+16 \beta\right), R_{2}=\frac{3 \alpha}{320 R_{0}^{2}}\left(3 \alpha^{2}+16 \beta\right), \ldots$ How ever, in the special case in which $\left(3 \alpha^{2}+16 \beta\right)=0$, that is $\beta=-\frac{3 \alpha^{2}}{16}$, we find that $R_{I}=0, I>1$.

\section{References}

1. A. Banerjee, S.B. Duttachoudhury, B.K. Bhui, Phys. Rev. D 40, 670 (1989)

2. K. Mosani, D. Dey, P.S. Joshi, Phys. Rev. D 101, 044052 (2020)

3. A. Banerjee, S. Chatterjee, N. Dadhich, Mod. Phys. Lett. A 35, 2335 (2002)

4. A. Banerjee, S. Chaterjee, Astrophys. Space Sci. 299, 3 (2004)

5. K.S. Govinder, M. Govender, Gen. Relativ. Gravit. 44, 147 (2012)

6. N.F. Naidu, M. Govender, S.D. Maharaj, Eur. Phys. J. C 78, 48 (2018)

7. J. Ospino, L.A. Nunez, Eur. Phys. J. C 80, 166 (2020)

8. S.C. Jaryal, Eur. Phys. J. C 80, 683 (2020)

9. W.B. Bonnor, A.K. de Oliveira, N.O. Santos, Phys. Rep. 181, 269 (1989)

10. B.V. Ivanov, Gen. Relativ. Gravit. 44, 1835 (2012)

11. R.A. Sussman, Class. Quantum Gravity 10, 2675 (1993)

12. P.C. Vaidya, Proc. Ind. Acad. Sci. A33, 264 (1951)

13. N.O. Santos, MNRAS 216, 403 (1985)

14. A.R. Liddle, R.J. Scherrer, Phys. Rev. D 59, 023509 (1999)

15. J.P. Uzan, Phys. Rev. D 59, 123510 (1999)

16. G.W. Bluman, S. Kumei, Symmetries and Differential Equations (Springer, New York, 1989)

17. T. Christodoulakis, N. Dimakis, P.A. Terzis, J. Phys. A Math. Theor. 47, 095202 (2014)

18. R. Mohanlal, S.D. Maharaj, A.K. Tiwari, R. Narain, Gen. Relativ. Gravit. 48, 87 (2016)

19. M.C. Kweyama, K.S. Govinder, S.D. Maharaj, Class. Quantum Gravity 28, 105005 (2011)

20. G. Abebe, S.D. Maharaj, K.S. Govinder, Eur. Phys. J. C 75, 496 (2015) 
21. M. Tsamparlis, A. Paliathanasis, Symmetry 10, 233 (2018)

22. V.V. Morozov, Classification of six-dimensional nilpotent Lie algebras. Izvestia Vysshikh Uchebn Zavendenii Matematika 5, 161 (1958)

23. G.M. Mubarakzyanov, Izvestia Vysshikh Uchebn Zavendeniı̌ Matematika 32, 114 (1963)

24. G.M. Mubarakzyanov, Izvestia Vysshikh Uchebn Zavendeniı̌ Matematika 34, 99 (1963)

25. G.M. Mubarakzyanov, Izvestia Vysshikh Uchebn Zavendeniǔ Matematika 35, 104 (1963)

26. M.J. Ablowitz, A. Ramani, H. Segur, Lettere al Nuovo Cimento 23, 333 (1978)

27. M.J. Ablowitz, A. Ramani, H. Segur, J. Math. Phys. 21, 715 (1980)

28. M.J. Ablowitz, A. Ramani, H. Segur, J. Math. Phys. 21, 1006(1980)

29. A. Paliathanasis, P.G.L. Leach, Int. J. Geom. Methods Mod. Phys. 13, 1630009 (2016)

30. S.D. Maharaj, M. Govender, IJMP D 14, 667 (2005)

31. R. Maartens, Causal thermodynamics in relativity (1996). arXiv:astro-ph/9609119v1

32. M. Govender, S.D. Maharaj, R. Maartens, Class. Quantum Gravity 15, $323(1998)$
33. J. Martinez, Phys. Rev. D 53, 6921 (1996)

34. S.M. Wagh, M. Govender, K.S. Govinder, S.D. Maharaj, P.S. Muktibodh, M. Moodley, Class. Quantum Gravity 18, 2147 (2001)

35. M. Govender, R.S. Bogadi, S.D. Maharaj, Int. J. Mod. Phys. D 26, 1750065 (2017)

36. A. Di Prisco, L. Herrera, M. Esculpi, Class. Quantum Gravity 13, 1053 (1996)

37. A. Di Prisco, N. Falcón, L. Herrera, M. Esculpi, N.O. Santos, Gen. Relativ. Gravit. 29, 1391 (1997)

38. J. Fort, J.E. Llebot, J. Phys. A Math. Gen. 29, 3427 (1996)

39. M. Govender, K.S. Govinder, Phys. Lett. A 283, 71 (2001)

40. L. Herrera, Phys. Rev. D 101, 104024 (2020) 